\title{
Capture of Low Grade Methane from Nitrogen Gas Using Dual Reflux Pressure Swing Adsorption
}

Thomas L. Saleman ${ }^{1}$, Gang (Kevin) $\mathrm{Li}^{1}$, Thomas E. Rufford ${ }^{1 \dagger}$, Paul Stanwix ${ }^{1}$, K. Ida Chan ${ }^{2}$, Stanley H. Huang $^{2}$, Eric F. May ${ }^{1} *$

1. Centre for Energy, School of Mechanical \& Chemical Engineering, The University of Western Australia, Crawley WA 6009, Australia

2. Chevron Energy Technology Company, Houston, TX 77002, USA.

Email: eric.may@uwa.edu.au

\begin{abstract}
We report a dual-reflux pressure swing adsorption (DR-PSA) apparatus and cycle configuration to recover an enriched methane product from mixtures of methane and nitrogen containing between (2.4 and 49.6) mol\% methane. This range of feed gas compositions is representative of some significant greenhouse gas emissions streams containing methane, including vent streams from liquefied natural gas production facilities and ventilation air from coal mining operations. The DR-PSA apparatus was demonstrated with activated carbon Norit RB3 as the adsorbent, operating with a low pressure step of 1.4 bar and a high pressure step of 5 bar. The effect of light reflux flowrate and heavy product draw on methane recovery and nitrogen vent purity were investigated. The DR-PSA experiment with 2.4 mol\% methane in the feed produced a methane product containing 35.7 mol\% methane, which is approximately a 15 times enrichment, and a clean nitrogen vent containing just 3000 ppmv methane. The capture of dilute methane with this DR-PSA process is energetically selfsustainable.
\end{abstract}

Keywords: greenhouse gas, methane emission, dual reflux pressure swing adsorption, nitrogen methane separation

\footnotetext{
${ }^{\dagger}$ Present address: School of Chemical Engineering, The University of Queensland, St Lucia Queensland 4072, Australia
} 


\section{Introduction}

Methane has a global warming potential 21 times higher than that of carbon dioxide and accounts for $16 \%$ of global greenhouse gas emissions (carbon dioxide equivalent) [1]. Significant sources of methane emissions include waste streams and fugitive emissions from oil and gas production facilities, ventilation air methane from coal mining activities, and agricultural activities such as cattle for dairy and beef production. The removal of methane from industrial waste gases is challenging as most often these waste streams are produced at low pressures and contain only small concentrations of methane; for example, ventilation air methane from a coal mine typically contains less than $1.5 \%$ methane [2]. The most cost-effective and practical technologies currently available to remove low concentrations of methane from low pressure gas streams are regenerative thermal oxidation and catalytic thermal oxidation processes that convert methane to carbon dioxide and water. Although some oxidation processes have potential for waste heat recovery, these processes typically present an additional cost to production. Hence, there is a clear motivation for the development of a methane recovery process that either returns methane to the sales gas stream or utilises the recovered methane for efficient power generation, resulting in lower operating costs and improved environmental outcomes. Adsorption-based processes including pressure swing adsorption (PSA) are potential technologies that could be used to recover methane from vent streams and enrich the methane to a concentration level that could be practically used in a lean-gas turbine or methane fuel cell [3-7]. In this work we demonstrate a dualreflux PSA (DR-PSA) process to recover, and enrich, methane from a low concentration methane and nitrogen mixture.

Pressure swing adsorption is an established technology employed to separate gas mixtures [8] by exploiting differences in the adsorption capacities of the constituent gases, or, in a few industrial examples, by exploiting differences in sorption rates to achieve a kinetic selectivity. A continuous 
adsorption process can be realised with multiple beds operating in cycles of high-pressure adsorption steps and low-pressure regeneration or desorption steps together with intermediate bed pressurisation steps. Such a stripping-type PSA process can be designed by selecting an appropriate adsorbent, operating pressures, flow regime and step durations to remove methane from nitrogen or air to a relatively low concentration, e.g. reducing from $55.2 \mathrm{~mol} \%$ to $11 \mathrm{~mol} \%$ with activated carbon [4]. However, one limitation in the application of PSA to gas purification is that few PSA designs can produce both a high-purity light product (raffinate) from the adsorption steps and an enriched secondary product (extract) during the regeneration steps. This constraint (often called a thermodynamic limit) [8-10] is strongly related to the ratio of the high and low pressures used in the process, and several vacuum PSA designs have been reported as one approach to enhance the enrichment of the secondary product [4, 11-14]. Other groups have investigated more complex PSA cycles featuring larger numbers of adsorption beds, intermediate recycle steps and pressure-balancing steps [7, 12, 15]. However, these more complicated PSA cycles require either increased numbers of adsorption beds or increased recycling of purified gas to regenerate the beds, leading to increased equipment and operating costs. There have been no feasibility studies performed with the inverse process known as enriching (or rectifying) PSA, although a theory of the process has been established [16] and it has been used to enrich trace components from air [9] with high enrichment ratios and recoveries.

The DR-PSA processes (also known as duplex PSA) studied by Diagne et al. [10, 17, 18], Ritter and co-workers [19, 20], Webley and Kearns [21], Thakur et al. [22] and Spoorthi et al. [23] combined the stripping and enriching PSA cycles into a single two-bed system, where the feed gas is fed to an intermediate position along the adsorbent bed and some of each product stream is refluxed into the respective ends of the two adsorption columns. Recently Bhatt et al. have performed an experimental investigation of DR-PSA with a split-column design, where each adsorbent bed is separated into two 
columns in series [24]. DR-PSA processes have features similar to a continuous distillation process; for example when both gas species are competing for adsorption sites the phase equilibria (vapor-solid) is akin to vapor-liquid equilibria [23], and the use of heavy (extract) and light (raffinate) refluxes through the adsorbent beds is similar to the use of liquid and vapor refluxes in a distillation process. In this paper we demonstrate experimentally a DR-PSA process to separate methane and nitrogen mixtures into a high-purity nitrogen stream, which can be safely vented to the atmosphere, and simultaneously an enriched methane product.

\section{Description of Laboratory Pressure Swing Adsorption Apparatus}

We designed and constructed a fully automated laboratory-scale pressure swing adsorption apparatus that can be operated in wide range of configurations to study both conventional PSA cycles and DRPSA cycles. The apparatus consists of two stainless steel adsorption columns $(3.5 \mathrm{~cm}$ internal diameter, $97 \mathrm{~cm}$ length), two mass spectrometers (MS) for gas composition analysis, pressure and flow control instrumentation, temperature indicators, recycle gas compressor, a $15 \mathrm{~L}$ feed mixing tank, a $12 \mathrm{~L}$ purge tank, a $12 \mathrm{~L}$ product tank, and data acquisition components. A simplified process flow diagram of the apparatus is presented in Figure 1 and photographs of the apparatus are shown in Figure 2. A general description of the apparatus is provided in this section while a detailed description of the experimental conditions used for the methane and nitrogen separations is given in Section 3.

The feed gas can enter at any of seven points along the length of the beds via seven pairs of valves located between the columns (only the middle pair of valves is shown in Figure 1). The middle pair of feed valves is located in the exact center of the two columns with the others valves spaced at $125 \mathrm{~mm}$ intervals, and the first and last valve pairs being $110 \mathrm{~mm}$ from the ends of each bed. Feed gas can also be supplied through a valve at the bottom of the feed manifold to allow operation of the apparatus in a 
conventional PSA mode. The feed flowrate and composition are controlled by three independent mass flow controllers (MFC) for each pure gas (nitrogen, methane and carbon dioxide, which was not used in this study) and mixed in a $15 \mathrm{~L}$ tank to dampen any composition or pressure fluctuations. Total flowrate, pressure and temperature of the feed are measured before the gas reaches the column manifold. The pressure in each column is controlled with back pressure regulators (BPRs 1 and 3) located at the top and bottom of the high and low pressure columns, respectively. The product and recycle flows are controlled by setting the heavy product draw with MFC5, which is the smaller of the two product flows for feeds containing low methane concentrations, and the light reflux flow with MFC4. We found this control strategy provided very stable operation during these experiments.

Although the low and high-pressure columns alternate through each DR-PSA cycle and the flow direction is also reversed, the heavy product is always drawn from the bottom of the apparatus and the light gas from the top of the apparatus. Both the heavy and light product lines include a 12 L purge tank to store purge gas and dampen pressure or composition fluctuations, but these purge tanks can be bypassed to reduce the time taken to transfer gas between the columns from several minutes to a few seconds.

Gas compositions are measured with two QMS-100 series mass spectrometers (MS) from Stanford Research Systems, which are connected to the two product gas streams in the system. A second set of $12 \mathrm{~L}$ storage tanks are available (not shown in Figure 1) to store light and heavy products for measurement of average compositions over a DR-PSA cycle, and they can be bypassed for the measurement of instantaneous compositions. Each MS was calibrated to have a maximum uncertainty of 0.005 in the mole fraction composition over the full range for binary mixtures of methane and nitrogen. We also measured carbon dioxide, oxygen, argon and water with these MS to check for back flow of air, which could potentially occur when there is no product flow because of the design of the 
MS. Check valves were installed to prevent the back-flow of air into the DR-PSA apparatus through the analysis lines.

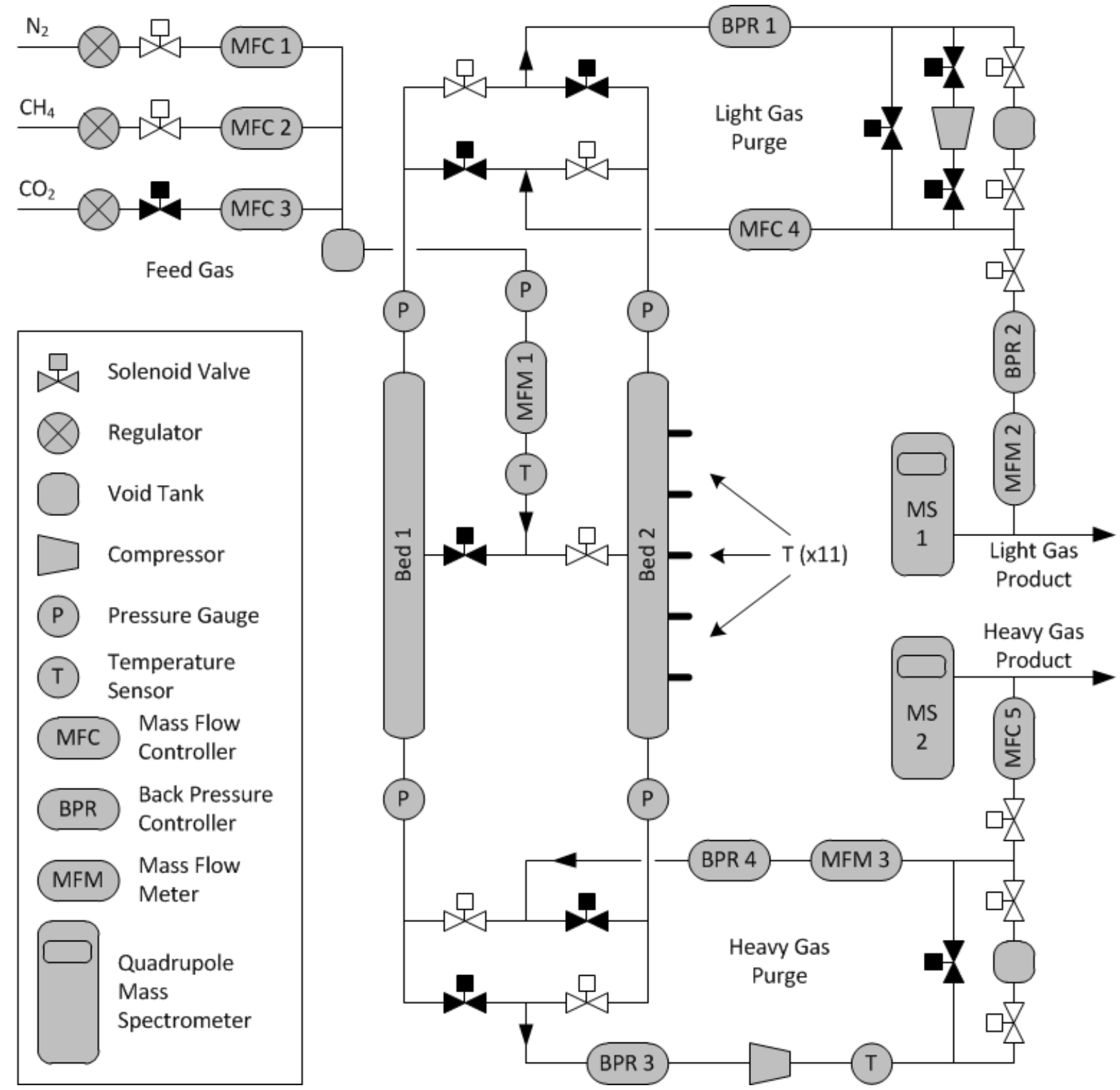

Figure 1. The simplified process flow diagram of the pressure swing adsorption apparatus. The valve positions (white $=$ open, black = closed) show the setup of a typical step of feed $/$ light purge for bed 2 with simultaneous heavy purge for bed 1 . For clarity details of some manual valves, additional flow lines, and the gas sampling system are not shown in this schematic. There are also eleven temperature sensors embedded along the length of each bed. 

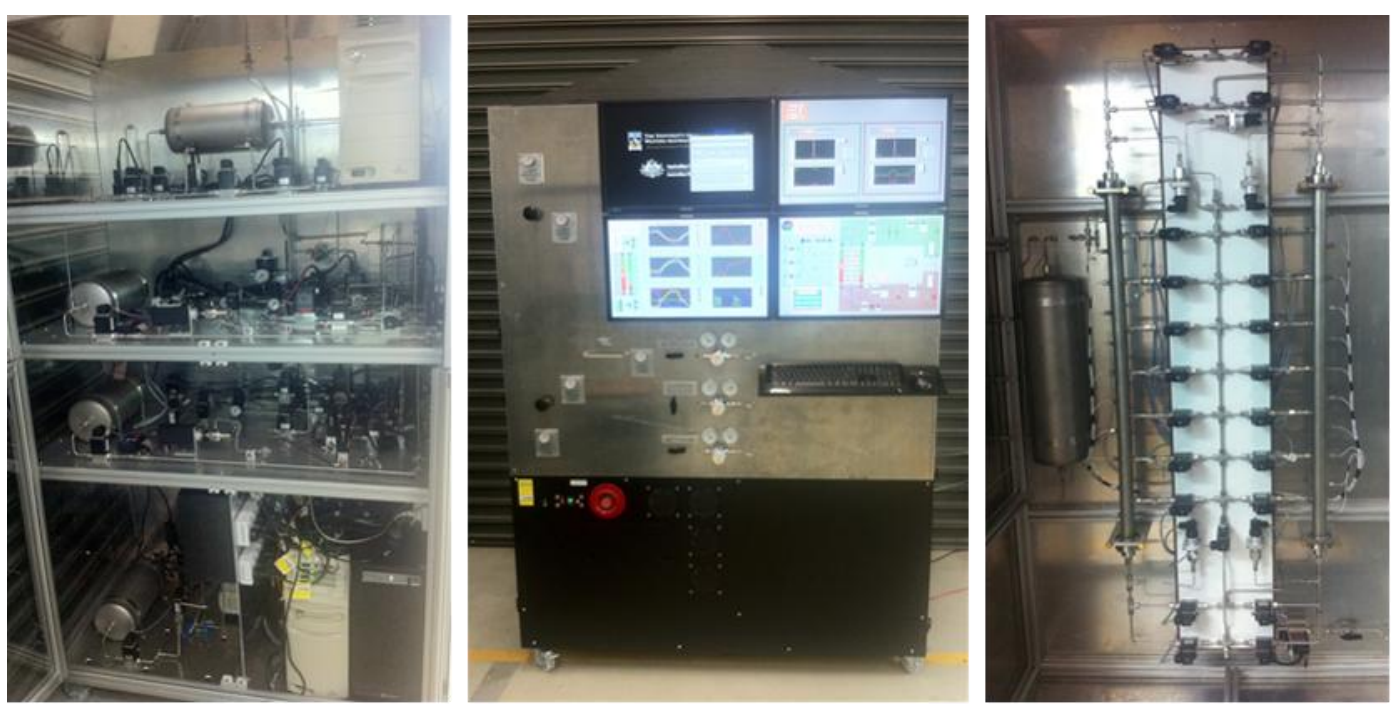

Figure 2. From left to right: Photos of the process instrumentation, control interface, and columns, in the DR-PSA apparatus.

The back pressure regulators (BPRs), mass flow meters (MFMs) and mass flow controllers (MFCs) were all sourced from Alicat Scientific Inc. and the uncertainties of the BPRs, MFCs and MFMs are $0.25 \%, 0.4 \%$ and $0.2 \%$ of the full scale, respectively, while the MFMs and MFCs have an additional uncertainty contribution of $0.8 \%$ of the measured flowrate. The MFMs and MFCs devices measure pressure drop along a laminar flow element within the device and use the Hagen-Poiseuille equation to calculate flowrate. This calculation requires knowledge of gas mixture's viscosity, which is dependent on the gas composition. In the DR-PSA experiments the composition of streams passing flow devices (MFM1, MFM3, MFC5, MFM2, MFC4) are variable and the measurement strategy employed was to set these devices to assume the flow was pure nitrogen during real-time operation. During postexperiment data processing, these apparent flowrates were corrected using the product compositions measured online. This correction is not required for the feed MFCs which only handle pure gases.

Four pressure transmitters (Bürkert, type $8324,5.3$ bar full scale) with an accuracy of $0.5 \%$ of the full scale, are used to measure the pressures at the top and bottom of each column and one in the feed line. Eleven temperature sensors (Omega PR-20 (PT100), Class A, 4-wire) are embedded along the length of 
each column to track the thermal front through the adsorbent beds. The middle temperature probe (labelled as T6) is located in the exact center of the column with the remaining probes spaced in intervals of $83 \mathrm{~mm}$, where the first and last probes are $70 \mathrm{~mm}$ from the ends of the column. Temperature sensors are also installed in the feed line and in the outlet of the heavy gas compressor.

\section{Experimental}

The DR-PSA apparatus was used to produce a high purity nitrogen raffinate and enriched methane extract from nitrogen and methane mixtures flowing at 1.25 SLPM and containing from (2.4 to 49.6) mol\% methane. This feed gas methane concentration range is representative of compositions typically found in LNG vent gas streams and coal mine ventilation gas, through to relatively concentrated methane in low grade natural gases.

Activated carbon Cabot Norit RB3 (from IMCD Australia Limited) was selected as the adsorbent in these DR-PSA experiments, since its equilibrium selectivity for methane from nitrogen of approximately 3.2 is typical of commercial adsorbents. Rufford et al. reported the equilibrium capacity and kinetic sorption measurements for methane and nitrogen on Norit RB3 [25]. The feed gas was supplied by Coregas with a stated purity of $99.995 \%$ and $99.999 \%$ for methane and nitrogen, respectively.

The DR-PSA was configured in a PL-A cycle as described by Kearns and Webley [21] with the feed supplied to the low pressure bed (PL) and the pressure reversal step (simultaneous pressurisation and blowdown) carried out with the strongly adsorbed gas species (conventionally labelled species A, which is methane in this study). Figure 1 shows the valve positions of the PL-A feed / purge step where Bed 1 is undergoing a high pressure purge while Bed 1 has a low pressure purge and simultaneous low pressure feed. In such 'A' cycles, the pressure is reversed through the heavy gas purge section (BPR 3, MFM 3, and BPR 4). The operating parameters held constant through all experiments are displayed in 
Table 1. Table 2 summarizes the 24 experimental runs performed at different feed compositions, heavy product draw rates and light reflux rates.

Each experiment was performed over approximately 24 hours, which corresponds to approximately 200 cycles (total cycle duration of 420 s), to allow the system to reach a cyclic steady state. We assessed several process parameters as indicators of cyclic steady state condition and found the best indicators to be the product compositions; Figure 3 shows the heavy product gas composition over the course of an entire experiment. Pressure and temperature profiles in the columns were not good indicators of cyclic steady state because the pressure reached a steady profile in just a few cycles and the temperatures changed only very slightly over a significant number of cycles. In addition, the ambient temperature fluctuations from day to night produced a small but noticeable drift in bed temperatures (up to $5 \mathrm{~K}$ ), further reducing the reliability of temperatures in determining the attainment of cyclic steady state.

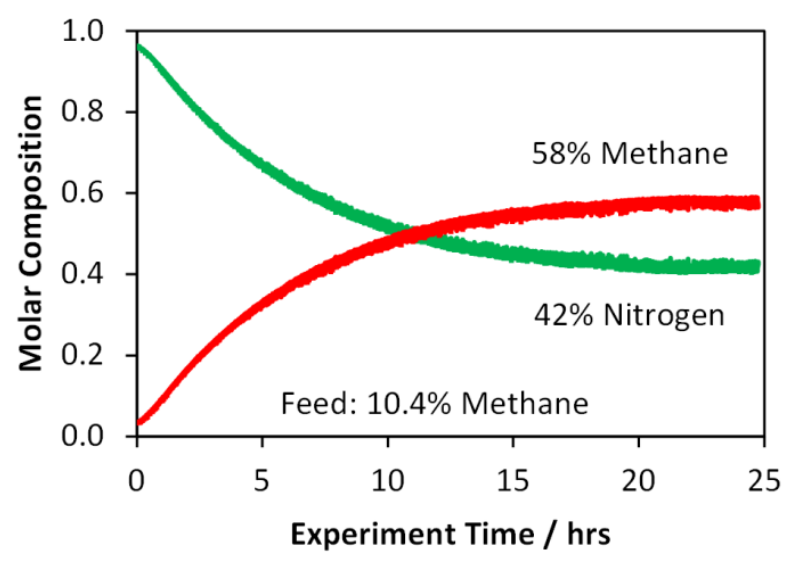

Figure 3. Evolution of the heavy product gas composition over the duration of an experiment. In this experiment (Run \#13 in Table 2) a 10.4\% methane feed mixture was enriched to 58\% methane in the heavy product while producing $96.7 \%$ pure nitrogen. Experiments typically started at the final conditions of the previous run to decrease the time taken for steady state to be achieved. 

Table 1 . Operating parameters
Figure 1 correspond to Step I.

\begin{tabular}{|c|c|c|c|}
\hline \multicolumn{4}{|c|}{ Feed and System Parameters } \\
\hline \multicolumn{2}{|c|}{ Feed flowrate } & \multicolumn{2}{|r|}{$1.25 \mathrm{SLPM}$} \\
\hline \multicolumn{2}{|c|}{ Feed pressure } & \multicolumn{2}{|r|}{$1.4 \mathrm{bar}$} \\
\hline \multicolumn{2}{|c|}{ Temperature (ambient) } & \multicolumn{2}{|r|}{$20-25^{\circ} \mathrm{C}$} \\
\hline \multicolumn{2}{|c|}{ High pressure } & \multicolumn{2}{|r|}{$5.0 \mathrm{bar}$} \\
\hline \multicolumn{2}{|c|}{ Low pressure } & \multicolumn{2}{|r|}{$1.4 \mathrm{bar}$} \\
\hline \multicolumn{2}{|c|}{ Pressure ratio } & \multicolumn{2}{|r|}{3.6} \\
\hline \multicolumn{2}{|c|}{ Fractional axial feed position } & \multicolumn{2}{|r|}{0.5} \\
\hline \multicolumn{4}{|c|}{ Adsorbent details } \\
\hline \multicolumn{2}{|l|}{ Material } & \multicolumn{2}{|l|}{ Norit RB3 } \\
\hline \multicolumn{2}{|c|}{ Pellet size } & \multicolumn{2}{|c|}{$\mathrm{D}: 3 \mathrm{~mm}, \mathrm{~L}: \sim 5 \mathrm{~mm}$} \\
\hline \multicolumn{2}{|c|}{ Mass loaded to each column } & \multicolumn{2}{|c|}{$410 \mathrm{~g}$} \\
\hline \multicolumn{2}{|c|}{ Bed voidage } & \multicolumn{2}{|l|}{0.433} \\
\hline \multicolumn{4}{|c|}{ Cycle Parameters } \\
\hline \multirow{5}{*}{$\begin{array}{l}\text { Step I } \\
\text { Step II } \\
\text { Step III } \\
\text { Step IV }\end{array}$} & Bed 1 & Bed 2 & Duration \\
\hline & HP Heavy Purge & LP Feed/Light Purge & $120 \mathrm{~s}$ \\
\hline & Blowdown & Pressurization & $90 \mathrm{~s}$ \\
\hline & LP Feed/Light Purge & HP Heavy Purge & $120 \mathrm{~s}$ \\
\hline & Pressurization & Blowdown & $90 \mathrm{~s}$ \\
\hline
\end{tabular}

Figure 4 and Figure 5 show pressure trends and flowrate trends, respectively, for a typical DR-PSA experiment that had reached cyclic steady state. Figure 4 also shows that there is a $35 \mathrm{kPa}$ and $12 \mathrm{kPa}$ pressure drop along the low and high pressure columns respectively, although this varied with the exact flowrate in each experiment. In the pressure trends, a few minor disturbances are observed during the feed and purge steps due to over-flows from the pressurization and blowdown steps. For example, at the start of the pressurization steps (Bed 1, Step IV and Bed 2, Step II) there is a large initial jump in pressure at the bottom of the column caused by sudden opening of a solenoid valve. In this instance 
there is up to a 1.5 bar pressure drop along the column which may impose a large shock to the adsorbent, and could potentially lead to attrition if the adsorbent were not tightly packed.

Figure 5 shows an example of the feed, light product, heavy product, light reflux and heavy reflux flowrates over a single cycle. The feed, heavy product and light reflux flowrates are maintained constant throughout the feed/purge steps (Steps 1 and 3). However, the heavy reflux flow has some large fluctuations at the start of the feed/purge steps due to the slow response of BPR4 (see Figure 1) and the flow characteristics of the compressor. During the pressure reversal step, the rate of gas transfer between the two columns exceeds the full range scale of flow meter MFM3, and thus the volume of gas transferred between the columns cannot be directly measured. Instead, this volume was independently calculated from the initial and final states of the columns. There is a slight variation in the total amount of light gas produced in each cycle as the back pressure regulator (BPR 2 in Figure 1) has some difficulty in controlling the start/stop nature of this flow; future work will aim to ensure this back pressure regulator in better tuned to remove this variation. However, this control limitation does not diminish the performance of the apparatus because it does not affect the flowrate out of the high pressure column or the flowrate into the low pressure column.

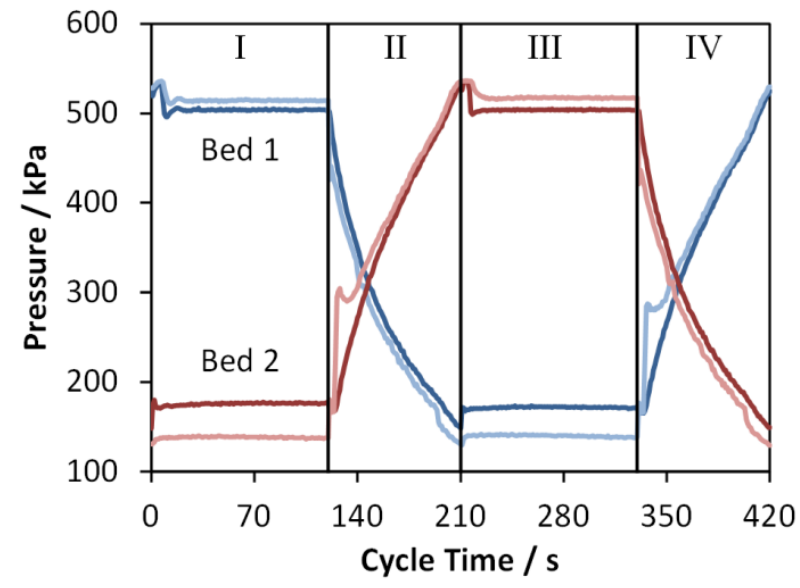

Figure 4. Column pressures over a single cycle operating with a heavy product draw of 0.229 SLPM and light reflux of 2.735 SLPM. The dark and light lines correspond to the top and bottom of the columns, respectively. 


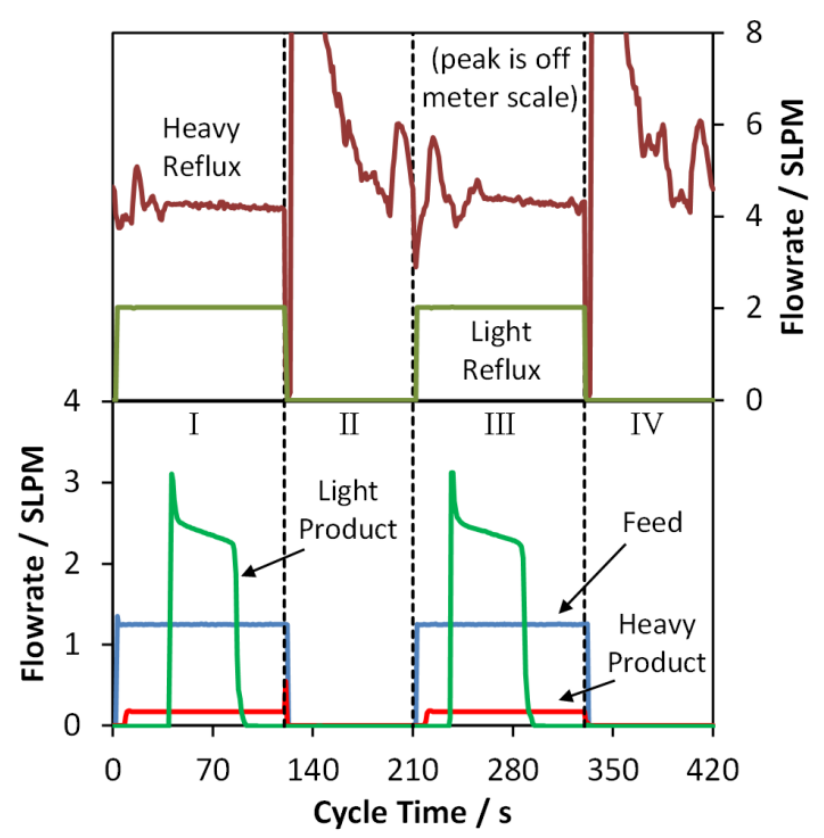

Figure 5. The measured flowrates for a single 4-step cycle, with the top and bottom panel corresponding to the internal and external flowrates, respectively. The feed (1.25 SLPM), heavy product (0.167 SLPM) and light reflux (1.992 SLPM) flowrates are controlled, and hence are constant over the feed/purge steps. Fluctuations in the heavy reflux are due to the slow response of BPR 4 (Figure 1), while the slight variation in the light product volume between Steps 1 and 3 is caused by the tuning of BPR 2.

\section{Results and Discussion}

Separation Performance. The results of the 24 experiments obtained at cyclic steady state (CSS) are summarized in Table 2 and presented in Figure 6. The overall mass balance for the experiments was within $5 \%$, with the majority being less than $1 \%$, which is within experimental uncertainty. Figure 6 illustrates the trade-off between the purity of nitrogen (light product) and methane (heavy product). The trend in product compositions is mostly independent of the methane concentration in the feed gas e.g. almost identical product purities of about $52 \mathrm{~mol} \%$ methane and $98.4 \mathrm{~mol} \%$ nitrogen from feeds containing (2.4 and 10.4) mol\% methane. Run 24 achieved the greatest methane enrichment ratio with 
the methane concentration increased from $2.4 \mathrm{~mol} \%$ to $51.3 \mathrm{~mol} \%$ in the heavy product, which is sufficiently rich to run in a lean combustion engine [26]. Experimental runs \#16, 17, 19, 20 and 21 all purified the methane-lean raffinate to $0.5 \mathrm{~mol} \%$ methane or less, which is substantially better than vent streams produced from conventional cryogenic distillation units for nitrogen rejection $\left(\sim 1.5 \mathrm{~mol} \% \mathrm{CH}_{4}\right)$ [27, 28]. Methane recovery to the heavy product was highest in Runs 1, 3, 5, 19 and 20. The experiments of Bhatt et al. [24] were conducted with a similar carbon adsorbent and a feed composition of $5 \mathrm{~mol} \% \mathrm{CH}_{4}$ and produced average $\mathrm{CH}_{4}$ and $\mathrm{N}_{2}$ products of $58 \mathrm{~mol} \%$ and $98 \mathrm{~mol} \%$ purity, respectively. The results of the separations reported by Bhatt et al. are also shown in Figure 6, and are broadly consistent with the results presented here. In this work, however, we systematically explored the separation of $\mathrm{N}_{2}$ and $\mathrm{CH}_{4}$ using DR-PSA cycles over a wide range of parameters for the purpose of (i) achieving significantly lower emissions in the raffinate $\left(>99.7 \mathrm{~mol}_{2} \mathrm{~N}_{2}\right)$ in Run $\# 16$ and \#19-21, (ii) obtaining highly enriched methane (> 16 times of enrichment ratio) in Run \#21 and 24, or (iii) the combination of both (Run \#21). These tests demonstrate the ability of the DR-PSA process to achieve a wide range of the target separation specifications.

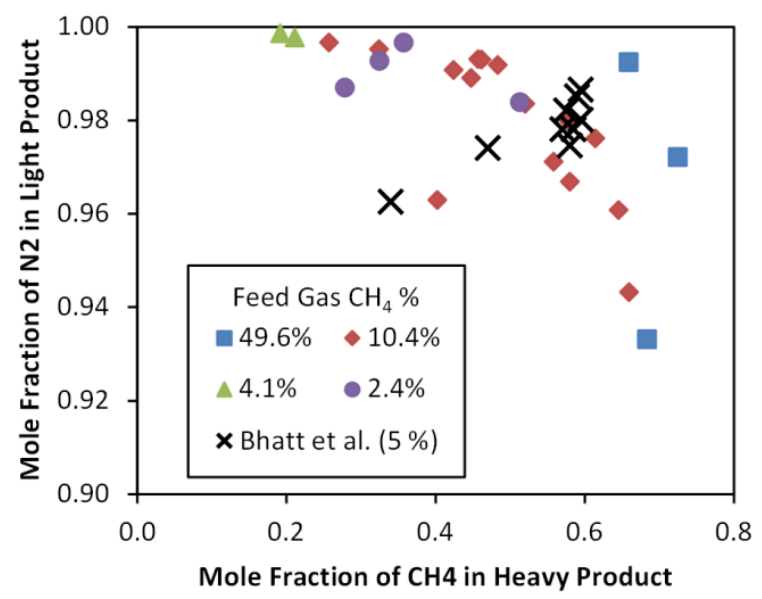

Figure 6. Separation performance map of $\mathrm{N}_{2}$ and $\mathrm{CH}_{4}$ mole fractions in the light and heavy products, respectively, obtained in 24 DR-PSA experiments with 1.25 SLPM feed of $\mathrm{CH}_{4}+\mathrm{N}_{2}$ mixtures at four $\mathrm{CH}_{4}$ concentrations. 
${ }_{4}^{3}$ Table 2. Summary of the feed, control parameters and results of all the DR-PSA experiments with nitrogen methane mixtures using the PL52 A cycle. All parameters that are not listed here are as displayed in Table 1. The specific work is an estimate of the compressor work required 63 per mole of a) feed gas treated and b) methane captured and has a relative uncertainty of about $30 \%$.

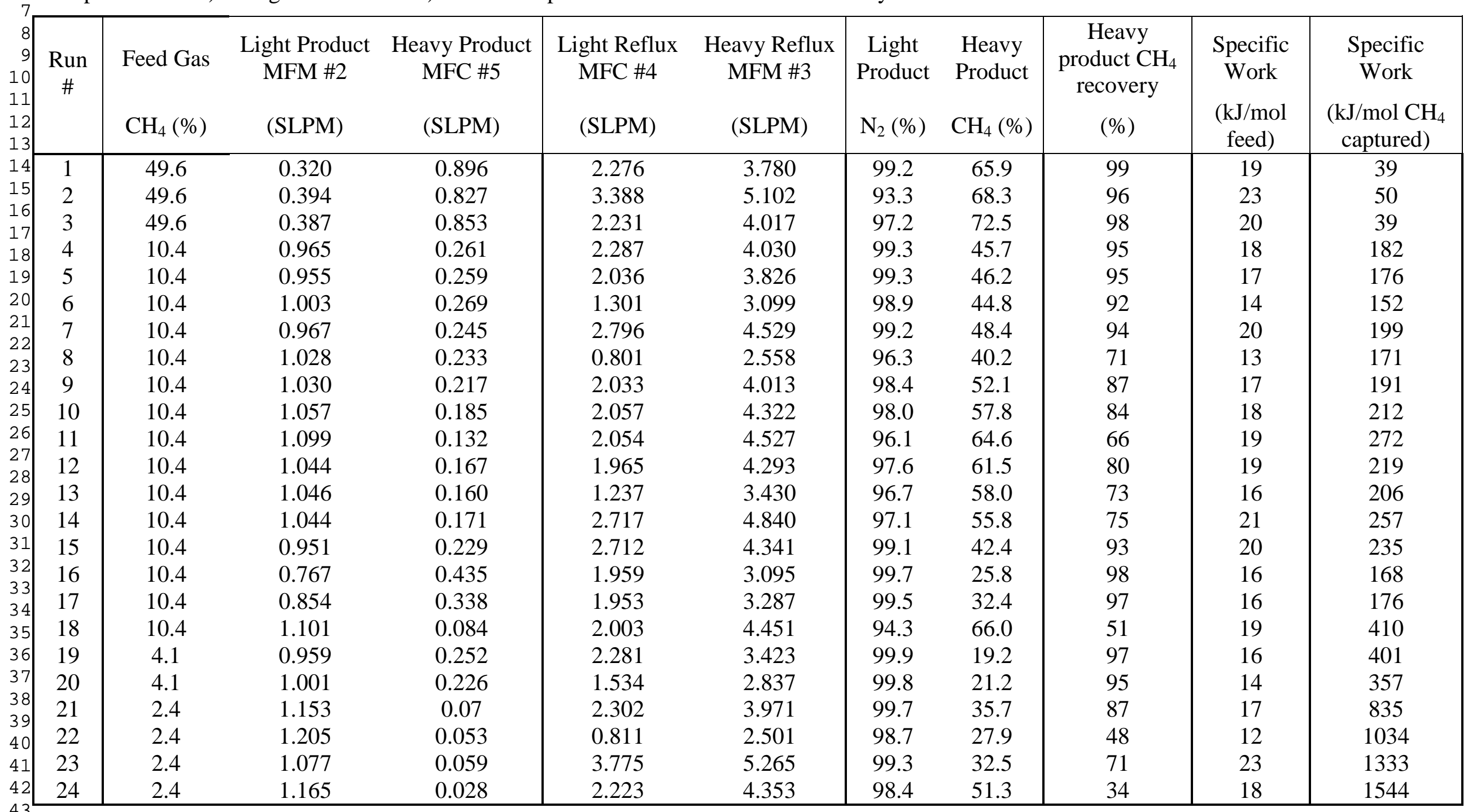


Energy Consumption. The work required to capture the methane is a key parameter in evaluating the sustainability and economics of the process. Figure 7 shows the compressor work done per mole of methane captured as a function of the mole fraction of $\mathrm{CH}_{4}$ in the feed for each of the 24 experimental runs. The minimum amount of capture work required decays exponentially with increasing methane concentration in the feed, although other the details of the cycle configuration, such as reflux and product flow rates, have a significant impact because they effect (i) the total work done per mole of feed and the (ii) the fraction of methane recovered in the heavy product. Nevertheless, for a feed composition of $2.4 \mathrm{~mol} \% \mathrm{CH}_{4}$, the minimum capture work is $835 \mathrm{~kJ}$ per mole of methane (Run \# 21), which is slightly lower than the standard heating value of methane, suggesting the potential for a marginal energy surplus. For methane feed concentrations higher than $4.1 \mathrm{~mol} \%$, the specific capture work is substantially lower than the power that can be generated using the captured methane (based on a typical 54-60\% energy efficiency for combined gas turbines) indicating the process is energetically self-sustainable; in particular, for a feed gas containing $10.4 \mathrm{~mol} \%$ methane, the energy available in the captured methane is more than five times the work required to collect it. Economically viable capture is readily conceivable even for feed concentrations below $2 \mathrm{~mol} \% \mathrm{CH}_{4}$ if either financial incentives are relevant and applicable (e.g. in jurisdictions where a price on $\mathrm{CO}_{2}$ equivalent emissions exist) and/or if an adsorbent with a higher equilibrium selectivity for $\mathrm{CH}_{4}$ over $\mathrm{N}_{2}$ is used. Under such circumstances, the capture of very dilute methane from large-scale industrial process flows, such as coal mine ventilation air $\left(\mathrm{CH}_{4}<1 \mathrm{~mol} \%\right)$ which represents a significant sources of greenhouse gas emissions [29], may become economically viable. 


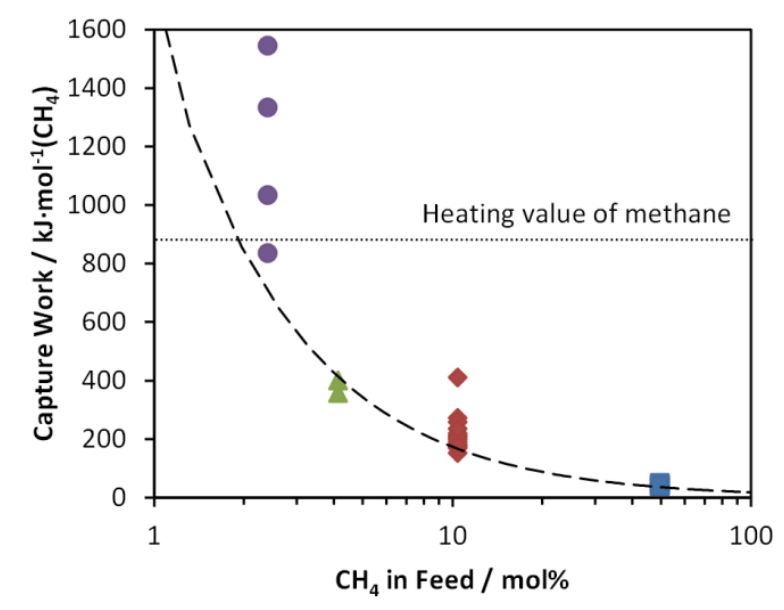

Figure 7. The specific work per mole of methane captured as a function of methane concentration in the feed to the DR-PSA unit, in comparison with the energy that can be generated using the captured methane for power or heating. Dash curve denotes a trend line of the minimum capture work at different feed concentrations.

Thermal Characteristics. Temperature waves in adsorption beds generally reflect the concentration profiles that develop in the column [30], so the axial temperature profiles shown in Figure 8 that developed in Bed 1 during the low pressure feed step (III) and the high pressure purge step (I) provide information about the $\mathrm{CH}_{4}$ concentration front during the DR-PSA cycles. Figure 8a shows the cooling of the bottom section of the low pressure column caused by desorption of $\mathrm{CH}_{4}$ into the combined flow of the $\mathrm{N}_{2}$-rich light reflux stream (entering the top of the column at fractional length $=1$, right hand side of Figure 8a) plus the feed gas (entering at fractional length $=0.5$ ). Most of the heavy gas produced from the bottom of the low pressure Column 1 in Step III is recycled to Column 2 as a heavy purge gas: only a small fraction - (0.6 to 20$) \%$ - of the $\mathrm{CH}_{4}$ enriched stream is drawn off as a heavy product. In the high pressure purge step (I) in Column 1 (Figure $8 \mathrm{~b}$ ) the $\mathrm{CH}_{4}$-enriched gas flows upwards through the bed causing $\mathrm{CH}_{4}$ to be adsorbed and a temperature wave to propagate towards the light end of the column associated with the enthalpy of $\mathrm{CH}_{4}$ adsorption. The temperature wave front is self-sharpening, reflecting the fact that methane's adsorption isotherm is 'favorable' [31] on activated 
carbon Norit RB3. With knowledge of the temperature wave fronts developed in the column, cycle structures can be engineered to prevent the fronts from reaching the end of the column to avoid contamination of the light product and maximize the utilization of the adsorbent's capacity. In these DR-PSA experiments $(10-60) \%$ of the purified $\mathrm{N}_{2}$ from the high pressure column in Step I is produced as raffinate and the other fraction is refluxed as the light purge gas to the low pressure column operating in the feed step (III).
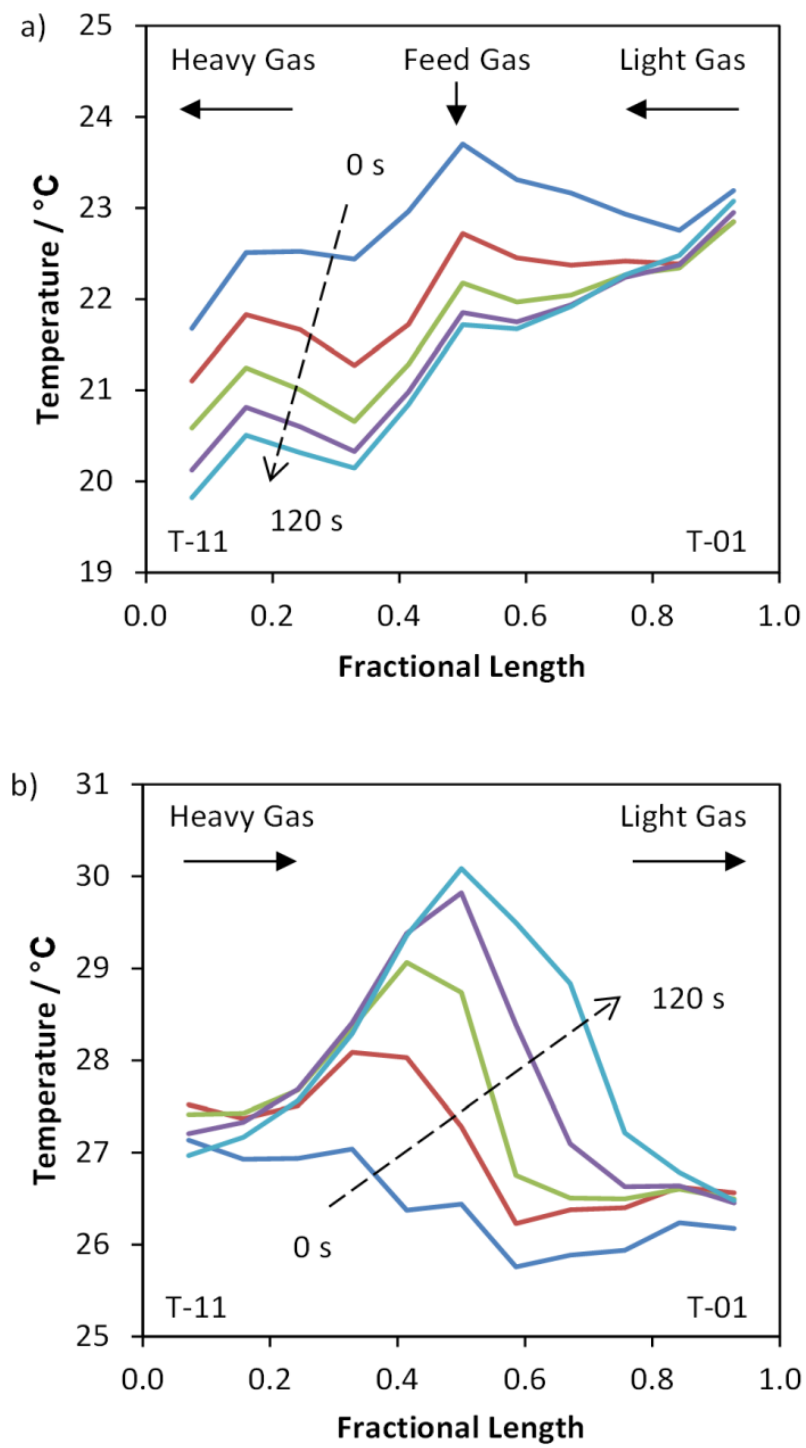
Figure 8. Development of temperature fronts in Column 1, Run \#7, during a) the low pressure feed Step III and b) the high pressure purge Step I. The plots show the progression of the adsorption and desorption fronts over $30 \mathrm{~s}$ intervals where a fractional length of zero indicates the bottom of the column.

Figure 9 shows the temperature at the top, middle and bottom of Column 1 over a single cycle at cyclic steady state. The largest variation occurs for $\mathrm{T}-06$ in the middle of the column $(\mathrm{z} / \mathrm{L}=0.500)$. The peak at the end of Step I corresponds to the peak at this position shown in Figure $8 \mathrm{~b}$ and is due to the methane adsorption front passing through the middle of the column around the end of the purge step. The peak-to-peak temperature variations experienced in the middle $(\mathrm{T}-06, \mathrm{z} / \mathrm{L}=0.5)$ and the bottom $(\mathrm{T}-11, \mathrm{z} / \mathrm{L}=0.072)$ of the column are similar in magnitude but the average temperature at the bottom of the column is lower. This is mainly caused by the regenerative heat exchange effect [32] where more cold stream flowed down during desorption (steps II and III) than the hot stream that flowed up during adsorption (steps IV and I). We suggest no net energy gain or loss across a full cycle at CSS, but there is a spatial misalignment (or hysteresis) between the exothermic adsorption thermal wave and endothermic desorption thermal wave. The temperature at the top of the column $(\mathrm{T}-01, \mathrm{z} / \mathrm{L}=0.928)$ varies to a much lesser degree than either the middle or the bottom because little methane adsorption occurs in this region, and the enthalpy of adsorption for $\mathrm{N}_{2}$ is smaller than for $\mathrm{CH}_{4}$.

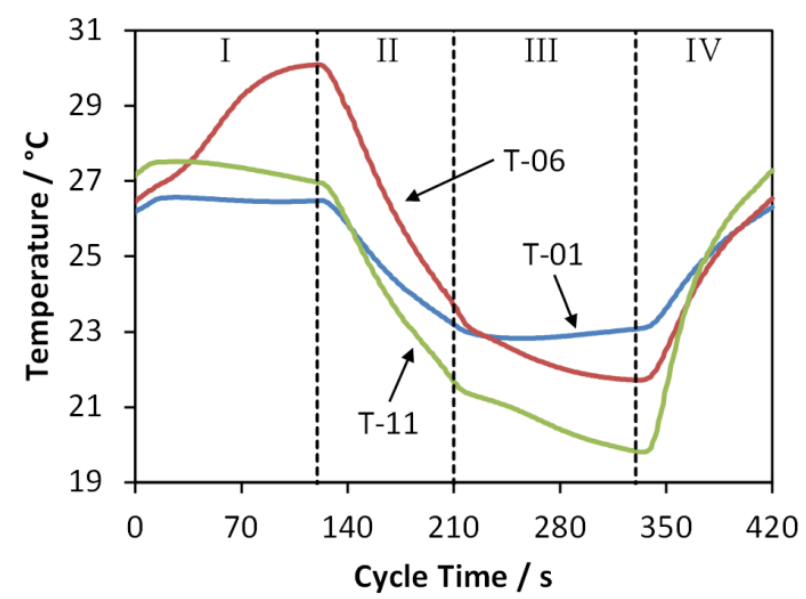


Figure 9. The temperature at the top, middle, and bottom of column 1 over a 4 -step steady-state cycle in Run 7. The fractional position of the three sensors are 0.928 (T-01), 0.500 (T-06), and 0.072 (T-11) where zero is the bottom of the column.

Effect of Light Reflux Flowrate. Experiments \#5, \#6, \#7 and \#8 with $10.4 \% \mathrm{CH}_{4}$ in the feed investigated the effect of increasing the light reflux flowrate from $(0.810-2.799)$ SLPM on the position of the $\mathrm{CH}_{4}$ composition fronts within the columns. This range of light reflux flow rates corresponds to a light reflux to feed ratio range of $(0.648-2.239)$. The changes in the temperature profile of Column 1 , as shown in Figure 10, during Step I and III provide an indication of the $\mathrm{CH}_{4}$ composition front's location. An increase in light reflux to the low pressure feed column is accompanied by an almost equal volume increase in heavy reflux to the high pressure column because the control scheme forces the heavy product flowrate to be constant. The temperature profiles developed in the HP heavy purge (Step I) and shown in Figure 10a, indicate that at higher light reflux flowrates more $\mathrm{CH}_{4}$ is adsorbed in the high pressure column, and the adsorption front propagates further into the bed. Consequently, a higher purity $\mathrm{N}_{2}$ light product $\left(99.2 \%\right.$ ) can be produced with a greater recovery of $\mathrm{CH}_{4}$ by operation of this DR-PSA cycle at a light reflux flowrate of 2.799 SLPM relative to 0.810 SLPM. The temperature profiles in Figure 10b recorded during the other half-cycle, in which the adsorbent is regenerated by the low pressure feed/light purge, highlight that a threshold light reflux ratio is necessary to ensure adequate desorption of $\mathrm{CH}_{4}$ from the bed and continuously produce high purity $\mathrm{N}_{2}$ from the low pressure column. For example, in experiment \#8, which was performed at the lowest reflux flowrate, there was insufficient light purge to effectively clean the adsorbent at cyclic steady state, resulting in a $\mathrm{N}_{2}$ purity of just $96.3 \%$ from a $89.6 \% \mathrm{~N}_{2}$ feed gas. The reflux ratio also affected the degree of $\mathrm{CH}_{4}$ enrichment with heavy product $\mathrm{CH}_{4}$ concentrations increasing from $40.2 \%$ to $48.4 \%$ as the light reflux rate was increased from 0.81 SLPM to 2.799 SLPM. 

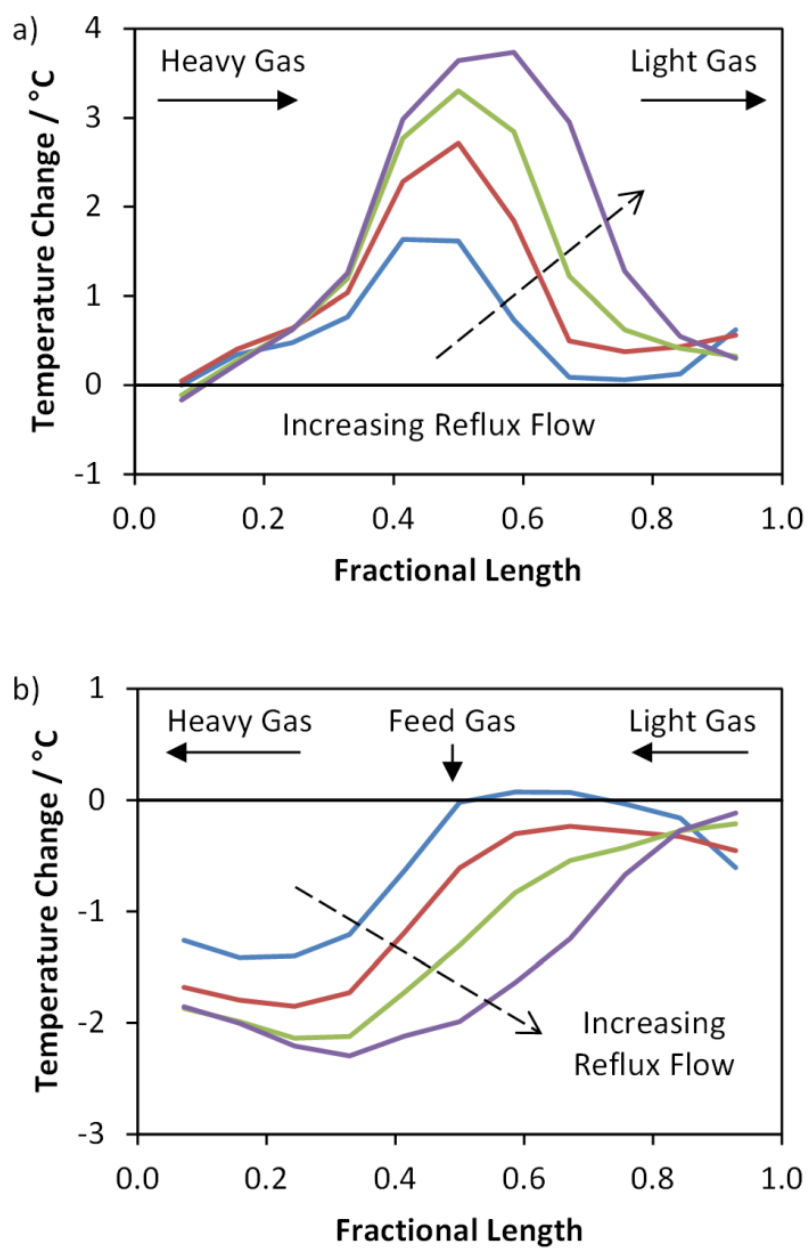

Figure 10. The effect of increasing the light reflux flowrate on the change in axial temperature through a) the high pressure bed during the heavy purge Step I and b) the low pressure bed during the feed Step III. The four traces correspond to light reflux flowrates of 0.810 (experiment \#8), 1.311 (experiment \#6), 2.046 (experiment \#5), and 2.799 (experiment \#7) standard liters per minute (SLPM), where the arrow indicates the impact of an increasing flowrate. 
Effect of Heavy Product Draw. Experiments \#5, \#10, \#11 and \#17 with $10.4 \% \mathrm{CH}_{4}$ in the feed investigated the effect of the heavy product flowrate in the range $(0.132-0.338)$ SLPM, which is equivalent to a heavy product to feed ratio of $(0.105-0.270)$, on the position of the $\mathrm{CH}_{4}$ composition fronts within the columns. The composition fronts are tracked from the change in the temperature profile of Column 1 over Steps I and III, as shown in Figure 11. The effect of increasing the heavy product draw is to shift the overall material balance towards a lower methane composition in the heavy product stream, making it easier to obtain high purity $\mathrm{N}_{2}$ in the light product stream. Figure 11a shows that when there is a low heavy product draw (0.132 SLPM, \#11) the $\mathrm{CH}_{4}$ propagates further up the column in the high pressure purge step; when desorption occurs this results in a highly enriched $\mathrm{CH}_{4}$ stream $(64.6 \%)$. However in this experiment, the $\mathrm{CH}_{4}$ breaks through the end of the bed and contaminates the light product, which results in a light product $\mathrm{N}_{2}$ purity of $96.1 \%$ and a $\mathrm{CH}_{4}$ recovery of only $66.5 \%$. In experiment \#17, the much larger heavy product draw of 0.338 SLPM, means that the $\mathrm{CH}_{4}$ only propagates half way along the bed; Figure $11 \mathrm{~b}$ shows that in this case, desorption of $\mathrm{CH}_{4}$ is significantly reduced so it is only enriched to $32.4 \%$. This experiment, however, produced a higher light gas $\mathrm{N}_{2}$ purity of $99.5 \%$ and had a $\mathrm{CH}_{4}$ recovery of $87.9 \%$. As the experiment is unable to achieve a perfect separation between the two components, the heavy product draw can be adjusted to shift the separation towards $\mathrm{CH}_{4}$ enrichment or $\mathrm{N}_{2}$ purification as required. 

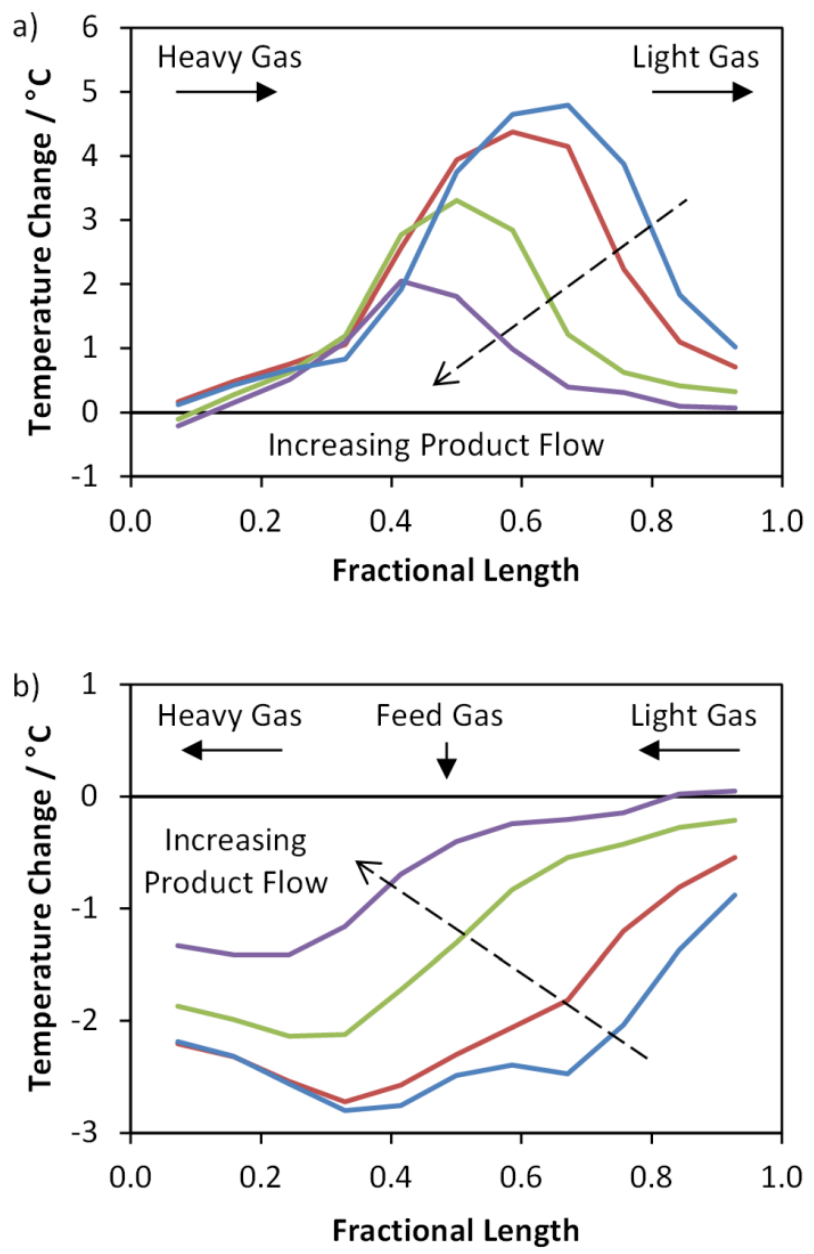

Figure 11. The effect of increasing the heavy product flowrate on the change in axial temperature through the a) high pressure purge step and b) low pressure feed step. The four plots correspond to a flowrate of 0.132 (\#11), 0.185 (\#10), 0.259 (\#5), and 0.338 (\#17) standard liters per minute, where the arrow indicates an increasing flowrate. Increasing the product flowrates reduces the reflux flowrates, hence why this has the opposite trend to Figure 10. 


\section{Conclusions}

A DR-PSA apparatus containing a standard activated carbon Norit RB3 as the adsorbent was used in a 4-step PL-A cycle to separate $\mathrm{CH}_{4}$ and $\mathrm{N}_{2}$ from $1.25 \mathrm{SLPM}$ of feed gas at $\mathrm{CH}_{4}$ compositions representative of industrial waste streams or low grade sources of natural gas. Under several sets of operating conditions the DR-PSA cycles produced $\mathrm{N}_{2}$-rich raffinate at $99.5 \%$ purity or better with $\mathrm{CH}_{4}$ enriched up to $65.9 \%$ in the heavy product gas and $\mathrm{CH}_{4}$ recoveries in excess of $95 \%$. The greatest $\mathrm{CH}_{4}$ enrichment factor of 21 was achieved for a feed gas containing $2.4 \% \mathrm{CH}_{4}$ to produce a heavy product with $51.3 \% \mathrm{CH}_{4}$, although this came with a relatively low $\mathrm{CH}_{4}$ recovery of $55 \%$. The effects of the light reflux and heavy product flowrates were tested and it was found that an increase of either of these flowrates caused an increase in $\mathrm{N}_{2}$ purity. However, the increased light gas purity achieved at increased heavy product flowrates was at the cost of reduced $\mathrm{CH}_{4}$ enrichment as dictated by the overall mass balance. The capture of dilute methane as low as $2.4 \mathrm{~mol} \%$ with DR-PSA process is energetically selfsustainable, and a five-fold energy return can be achieved for the capture from a $10.4 \mathrm{~mol} \%$ feed. These experiments demonstrate the potential of the DR-PSA technology to reduce $\mathrm{CH}_{4}$ emissions at a relatively low energy cost with potential applications in the recovery of low grade $\mathrm{CH}_{4}$ from coal mine ventilation air and the polishing of $\mathrm{N}_{2}$ vent streams generated during LNG production. Our future work will investigate the effect of cycle timing, feed port location, and explore other DR-PSA configurations presented by Kearns and Webley [21].

\section{Acknowledgement}

The authors thank C. Grimm for the construction of the apparatus and G.C.Y. Watson for input to the conceptual design of the apparatus. This research was sponsored by Chevron ETC and the Department of Environment Regulation of Western Australia through its Low Emissions Energy Development (LEED) Fund. G.L. is the recipient of an Australian Research Council Discovery Early Career Researcher Award (DE140101824). T.R. continues to study in gas separation processes at the University of Queensland as another recipient of an Australian Research Council Discovery Early Career Researcher Award (DE140100569). 


\section{References}

[1] IPCC, Climate Change 2014: Mitigation of Climate Change. Contribution of Working Group III to the Fifth Assessment Report of the Intergovernmental Panel on Climate Change, Cambridge University Press, Cambridge, United Kingdom and New York, NY, USA, 2014.

[2] I. Karakurt, G. Aydin, K. Aydiner, Mine ventilation air methane as a sustainable energy source, Renewable and Sustainable Energy Reviews 15 (2011) 1042-1049.

[3] A.I. Fatehi, K.F. Loughlin, M.M. Hassan, Separation of Methane-Nitrogen Mixtures by Pressure Swing Adsorption Using a Carbon Molecular-Sieve, Gas Sep Purif 9 (1995) 199-204.

[4] A. Olajossy, A. Gawdzik, Z. Budner, J. Dula, Methane separation from coal mine methane gas by vacuum pressure swing adsorption, Chem Eng Res Des 81 (2003) 474-482.

[5] K. Warmuzinski, L. Buzek, W. Sodzawiczny, M. Tanczyk, S. Smigasiewicz, Methane/nitrogen separation by pressure swing adsorption on a carbon molecular sieve, Inz Chem Procesowa 19 (1998) 915-929.

[6] J.A. Delgado, M.A. Uguina, J.L. Sotelo, B. Ruiz, Modelling of the fixed-bed adsorption of methane/nitrogen mixtures on silicalite pellets, Sep Purif Technol 50 (2006) 192-203.

[7] J.A. Delgado, M.A. Uguina, J.L. Sotelo, V.I. Agueda, P. Gomez, Numerical simulation of a three-bed PSA cycle for the methane/nitrogen separation with silicalite, Sep Purif Technol 77 (2011) 7-17.

[8] C.A. Grande, Advances in Pressure Swing Adsorption for Gas Separation, ISRN Chemical Engineering 2012 (2012) 13.

[9] M. Yoshida, J.A. Ritter, A. Kodama, M. Goto, T. Hirose, Enriching reflux and parallel equalization PSA process for concentrating trace components in air, Ind Eng Chem Res 42 (2003) 1795-1803.

[10] D. Diagne, M. Goto, T. Hirose, New Psa Process with Intermediate Feed Inlet Position Operated with Dual Refluxes - Application to Carbon-Dioxide Removal and Enrichment, J Chem Eng Jpn 27 (1994) 85-89.

[11] C.A. Grande, E. Basaldella, A.E. Rodrigues, Crystal size effect in vacuum pressure-swing adsorption for propane/propylene separation, Ind Eng Chem Res 43 (2004) 7557-7565.

[12] F.A. Da Silva, A.E. Rodrigues, Propylene/propane separation by vacuum swing adsorption using 13X zeolite, Aiche J 47 (2001) 341-357.

[13] L.T. Biegler, L. Jiang, V.G. Fox, Simulation and optimization of pressure-swing adsorption systems for air separation, Aiche J 49 (2003) 1140-1157.

[14] F.A. Da Silva, A.E. Rodrigues, Vacuum swing adsorption for propylene/propane separation with 4A zeolite, Ind Eng Chem Res 40 (2001) 5758-5774.

[15] A.E. Rodrigues, F.V.S. Lopes, C.A. Grande, Activated carbon for hydrogen purification by pressure swing adsorption: Multicomponent breakthrough curves and PSA performance, Chem Eng Sci 66 (2011) 303-317.

[16] A.D. Ebner, J.A. Ritter, Equilibrium theory analysis of rectifying PSA for heavy component production, Aiche J 48 (2002) 1679-1691.

[17] D. Diagne, M. Goto, T. Hirose, Experimental-Study of Simultaneous Removal and Concentration of Co2 by an Improved Pressure Swing Adsorption Process, Energ Convers Manage 36 (1995) 431-434.

[18] D. Diagne, M. Goto, T. Hirose, Parametric Studies on Co2 Separation and Recovery by a Dual Reflux Psa Process Consisting of Both Rectifying and Stripping Sections, Ind Eng Chem Res 34 (1995) 3083-3089.

[19] A.D. Ebner, J.A. Ritter, Equilibrium theory analysis of dual reflux PSA for separation of a binary mixture, Aiche J 50 (2004) 2418-2429.

[20] J.A. McIntyre, A.D. Ebner, J.A. Ritter, Experimental Study of a Dual Reflux Enriching Pressure Swing Adsorption Process for Concentrating Dilute Feed Streams, Ind Eng Chem Res 49 (2010) 1848-1858.

[21] P.A. Webley, D.T. Kearns, Modelling and evaluation of dual-reflux pressure swing adsorption cycles: Part I. Mathematical models, Chem Eng Sci 61 (2006) 7223-7233. 
[22] R.S. Thakur, N. Kaistha, D.P. Rao, Process intensification in duplex pressure swing adsorption, Comput Chem Eng 35 (2011) 973-983.

[23] G. Spoorthi, R.S. Thakur, N. Kaistha, D.P. Rao, Process intensification in PSA processes for upgrading synthetic landfill and lean natural gases, Adsorption 17 (2011) 121-133.

[24] T.S. Bhatt, A. Sliepcevich, G. Storti, R. Rota, Experimental and Modeling Analysis of Dual-Reflux Pressure Swing Adsorption Process, Ind Eng Chem Res 53 (2014) 13448-13458.

[25] T.E. Rufford, G.C.Y. Watson, T.L. Saleman, P.S. Hofman, N.K. Jensen, E.F. May, Adsorption Equilibria and Kinetics of Methane plus Nitrogen Mixtures on the Activated Carbon Norit RB3, Ind Eng Chem Res 52 (2013) 14270-14281.

[26] J. Kim, A. Maiti, L.-C. Lin, J.K. Stolaroff, B. Smit, R.D. Aines, New materials for methane capture from dilute and medium-concentration sources, Nat Commun 4 (2013) 1694.

[27] B.K. Lamb, J.B. McManus, J.H. Shorter, C.E. Kolb, B. Mosher, R.C. Harriss, E. Allwine, D. Blaha, T. Howard, A. Guenther, R.A. Lott, R. Siverson, H. Westburg, P. Zimmerman, Development of Atmospheric Tracer Methods To Measure Methane Emissions from Natural Gas Facilities and Urban Areas, Environ Sci Technol 29 (1995) 14681479.

[28] Nitrogen Rejection Unit Optimization, PRO Fact Sheet No. 905, US EPA, Partner Reported Opportunities (PROs) for Reducing Methane Emissions, 2011.

[29] C.Ö. Karacan, F.A. Ruiz, M. Cotè, S. Phipps, Coal mine methane: A review of capture and utilization practices with benefits to mining safety and to greenhouse gas reduction, International Journal of Coal Geology 86 (2011) 121-156.

[30] G. Li, P. Xiao, D. Xu, P.A. Webley, Dual mode roll-up effect in multicomponent non-isothermal adsorption processes with multilayered bed packing, Chem Eng Sci 66 (2011) 1825-1834.

[31] D.M. Ruthven, Principles of adsorption and adsorption processes, Wiley, New York, 1984.

[32] G. Li, P. Xiao, J. Zhang, P.A. Webley, D. Xu, The role of water on postcombustion CO2 capture by vacuum swing adsorption: Bed layering and purge to feed ratio, Aiche J 60 (2014) 673-689. 